\title{
Novel Optical Titration Sensor based on Integrated Planar Polymer Waveguides
}

Felix Betschon, Markus Halter, vario-optics ag, Mittelbissaustr. 7, 9410 Heiden, Switzerland

Markus Michler, Johannes Kremmel, University of Applied Sciences Buchs (NTB), Institute for Micro- and Nanotechnology, Werdenbergstr. 4, 9471 Buchs, Switzerland

Björn Christensen, Philipp Schraner, Metrohm AG, Oberdorfstr. 68, 9100 Herisau, Switzerland

\section{Abstract}

The present paper describes a novel optical titration sensor based on planar polymer waveguides integrated on a printed circuit board. An overview is given over the compact opto-electronic sensor. The design was done using ray-tracing software and realised with the planar polymer waveguide technology. For structuring the light guides a lithographic process was used which allows the cost-effective realization of very complex optical structures. The optical system consists of 8 light sources (LEDs) of different wavelengths, a coupling system based on planar polymer waveguides, which collects and couples the light into a free-space line. The light is collected and converted to a electrical signal by a photodiode. The whole system is integrated into a glass cuvette. The results of first sensor functionality tests prove the ability of the sensor module to determine a 4-acid mixture with only one indicator mixture in a single titration run. Furthermore, the speed can be set much faster than in a classical titration.

\section{Introduction}

One advantage of titration as an analytical method is that small quantities of a substance can be determined with inexpensive equipment (100 - 3000 EUR) in a short time (within a few minutes) with good accuracy and repeatability ( $1 \% \ldots 0.05 \%)$ [1]. Although visually indicated titrations are still needed e.g. prescribed in the pharmacopoeia, they have lost much of importance in laboratory automation. Major drawbacks of conventional optical methods include:

- the selection of the indicator requires a good knowledge of the chemical reaction

- the amount of the indicator influences the analysis result

- the colour change of an indicator must be suitable to the appropriate wavelength of light

- only one substance can be determined

These drawbacks are opposed to the advantage of a much faster tracking of the equilibrium by optical titration. Because of the above limitations various optical titration sensors are sold dedicated to special applications.

Multi-channel sensors are designed for spectral analysis and are very expensive $(>4000 €)$. These devices are not adequate for titration, since the measured data of such devices cannot be passed directly to a titration device. Cheaper products, which can measure only one channel, can be connected to the analog input of present titrators. But their signal transfer is analog and very slow. The disadvantage of all of these sensors is that they always measure with one wavelength and therefore only one substance can be determined.

Therefore, the goal of this project was to combine all the advantages of single channel titration in a miniaturized multi channel titration sensor using multiple light sources and optical combiners based on optical polymer waveguides, integrated on a printed circuit board. For this, the entire waveguide system, as well as the necessary driver electronics, had to be developed, manufactured and characterized. In addition, the opto-electronic sensor had to be integrated into a glass cuvette in order to be chemically inert and mechanically compatible to conventional glass electrodes.

\section{System Overview and Measuring Principle}

The sensor contains all the necessary components for light generation, light guiding and light detection (Fig. 1). Light of at least eight wavelengths is generated by commercially available semiconductor light sources. The electronic and optical components are completely encapsulated in glass. The optical signals are sent through the sample medium and detected on the opposite side. The measurement system allows the detection of changes in the light transmission properties of the sample at several wavelengths, almost simultaneously with a single detector. 
The LEDs and the photodiode are controlled by a microcontroller on the electro-optical circuit board $(E O C B)$. A USB port enables data transmission and the electrical supply. Because of this, the sensor can be operated by a standard PC. The EOCB is hermetically sealed in a glass shaft developed by Metrohm. Thus, the sensor is protected from aggressive media and provides mechanical compatibility with existing titration systems.
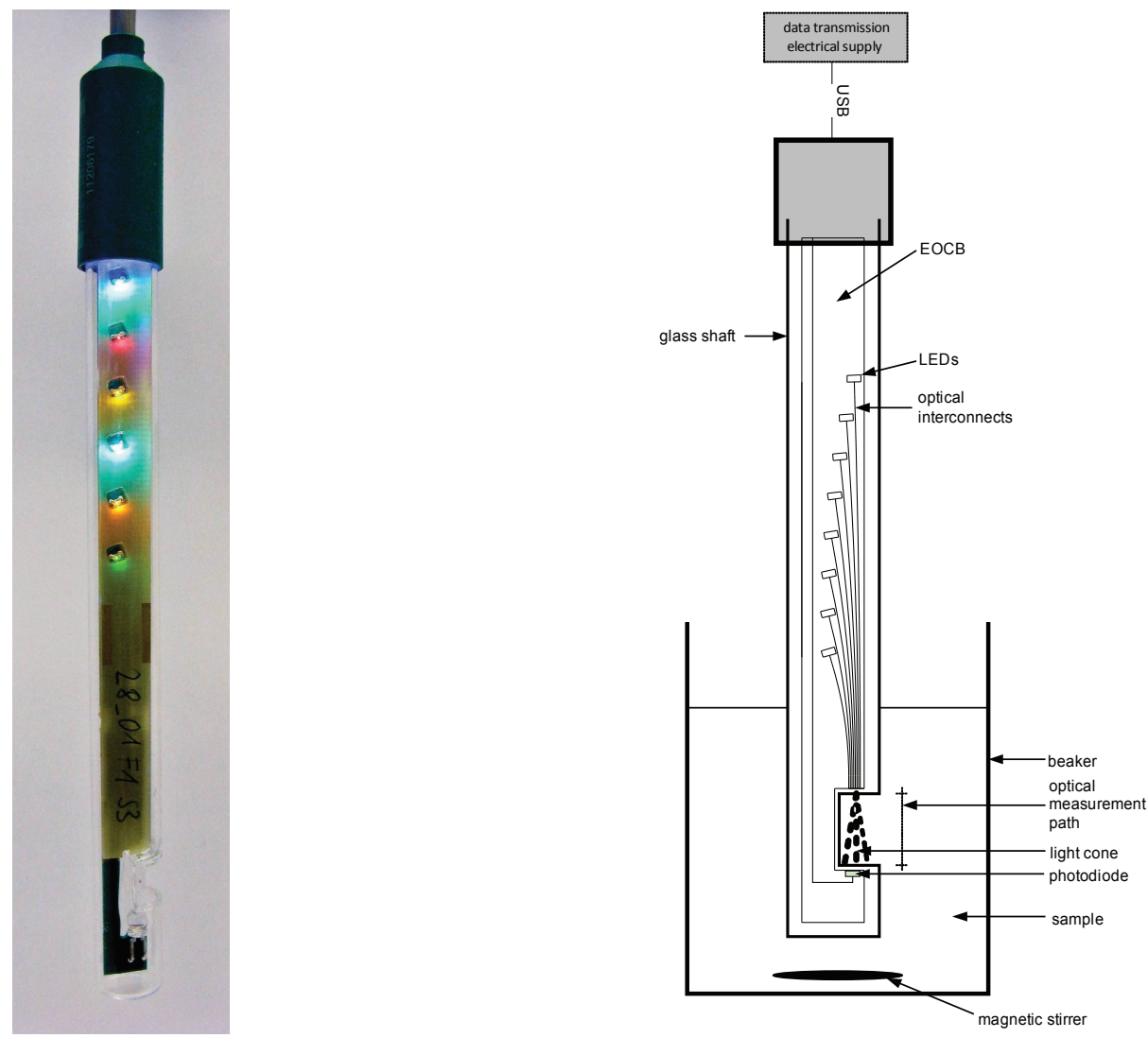

Fig. 1: a)Titration sensor, b) Principle build-up

In a sample, the relative signal change, which is caused by adding a defined amount of a chemical standard solution, is measured (colour change of an indicator). The signal change is plotted against the volume. This results in typical V- or S-shaped curves which are then evaluated with known algorithms.

The LEDs are modulated on a high frequency. The ambient light can be measured during phases where all LED's are switched off and can then be compensated automatically from the LED signals. With this method influences of the ambient light can almost completely be eliminated.

\section{Optical Design}

The light guide path of the present integrated optical sensor consists of eight LED light sources with different wavelengths coupled to combiner structures (for the combination of different wavelengths into a single path). For measurement purposes the light leaves the light guide, crosses a measurement section and finally strikes the detector (Fig. 2b). Output coupling structures optimize the irradiation pattern on the detector to increase the signal to noise ratio. We therefore investigated different kinds of functional structures, namely

- Coupling structures to optimize the coupling efficiency of LEDs to light guides

- Combiner structures

- Output coupling structures from light guide to free space coupling

Due to the large core diameters of the light guides, the propagation of light through the guides can be described by the laws of geometrical optics. Hence, the layout of the structures was performed using raytracing simulations and calculations (Zemax) 


\subsection{Coupling Structures}

When LEDs and light guides are coupled, the light can propagate only if it strikes the front surface of the core with an angle of incidence below the acceptance angle of the light guide. If the emitter is small compared to the light guide dimensions, the angle of incidence of light striking the outer regions of the core exceeds the acceptance angle most likely. Uematsu et al. and Hudson ([2], [3]) showed that in this case the coupling efficiency can be increased by applying a waveguide taper. With an appropriate taper the effective numerical aperture is increased by the taper ratio $r=a_{2} / a_{1}$ (see Fig. 2). But, due to the lithographic fabrication method waveguide tapering is limited to the patterned plane.

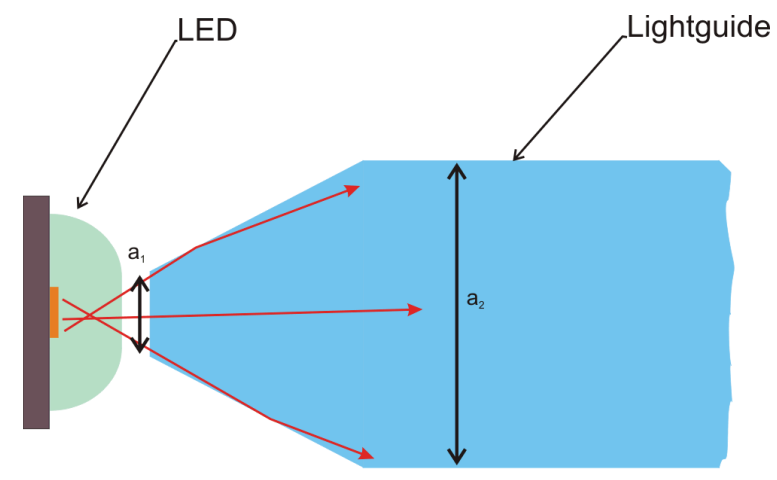

Fig. 2: Coupling of an LED to a light guide employing a coupling taper

\subsection{Combiner Structures}

Combiner and splitter structures belong to the basic structures of integrated optics. They are mainly used for combining light from different light sources or for the splitting of one single light path into different branches. Kurokawa et al. [4] showed that the losses in a multimode combiner structure mainly depend on the width of the outgoing light guide. The geometrical losses in an ideal combiner structure correspond to the ratio of the width of the outgoing light guide to the accumulated width of the incoming light guides. If the boundary conditions, light guide widths, angle or radii of the branches, are given and the propagating light uses the whole propagable angle-spectrum a further optimization is not possible. In the taperedsection the light guide shrinks from the double width of the input-light guides to the width of the output light guide, the Lagrange-Invariant $n^{*} a^{*} \sin (\theta)=$ const. causes the sines of the propagation-angles of the light to increase by the taper ratio [5]. The light propagating at angles that exceed the numerical aperture of the light guide is lost. Additional losses are caused by the angle or the radius of the two input branches (Fig. 3).

Due to the limited imaging properties of the exposure system, vertices of V-structures only develop improperly. These effects have to be considered during design and optimization in addition to the known geometrical aspects.

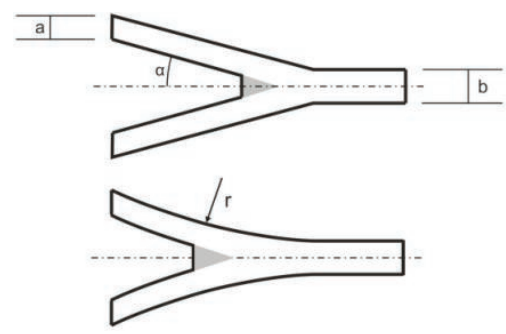

Fig. 3: Combiner structures with linear and curved branches

\subsection{Output Coupling Structures}

The presented sensor uses a free space section for measurement purposes. Therefore, the light has to be transferred from the light guide system through a free-space section onto a detector. To enhance the detector signal it is necessary to optimize the irradiance onto the detector. This is done by specific output coupling structures (Fig. 4). With a taper the cross section of the light guide is increased, while, due to the Helmholtz-Lagrange invariant the divergence of the radiation decreases. To optimize the irradiance pattern, lensed surfaces were applied. The taper geometry, as well as the lensed surfaces were optimized again using ray-tracing simulations. 


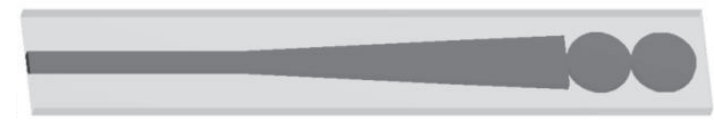

Fig. 4: Output coupling structure with taper and two cylindrical lenses

To evaluate the validity of the simulation results, sample structures were fabricated and characterized. The measurement results were in good agreement with the simulation results.

\subsection{Final Layout of the Waveguide Structure}

Based on the results of the evaluated functional structures the layout of integrated optical system of the sensor was designed (Fig. 5). It consists of eight LED sources with different wavelengths, a 8x1 combiner structure and an output-coupling structure to illuminate the photodiode through a $10 \mathrm{~mm}$ free-space section. To provide a sufficient resolution of the sensor, the eight LEDs were placed at the input light guides of the combiner in a way that the resulting photo currents are in the same order of magnitude.

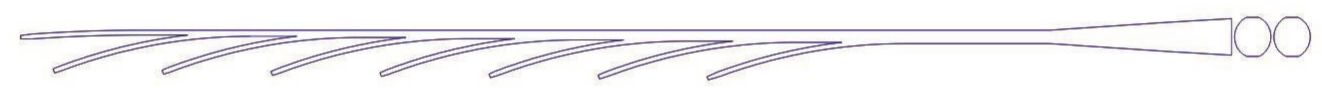

Fig. 5: Final layout of waveguide structure

\section{$5 \quad$ Manufacturing of Electro-Optical Circuit Board}

The optical structures were implemented on the printed circuit board using a planar polymer waveguide technology ([6], [7]). The structuring of the light guides was done using photolithography. This allows a cost-effective realization of the complex optical structures compared to other realization concepts (e.g. glass fibers).

\subsection{Layer Build-up}

The system consists of an electrical printed circuit board (PCB) and an optical board which is mounted upside down on the electrical circuit board. The reason for this "2-board" solution are limitations of the equipment used for the electronic components. In future versions the optical layer could be applied directly onto the electrical PCB and the additional substrate can be eliminated.

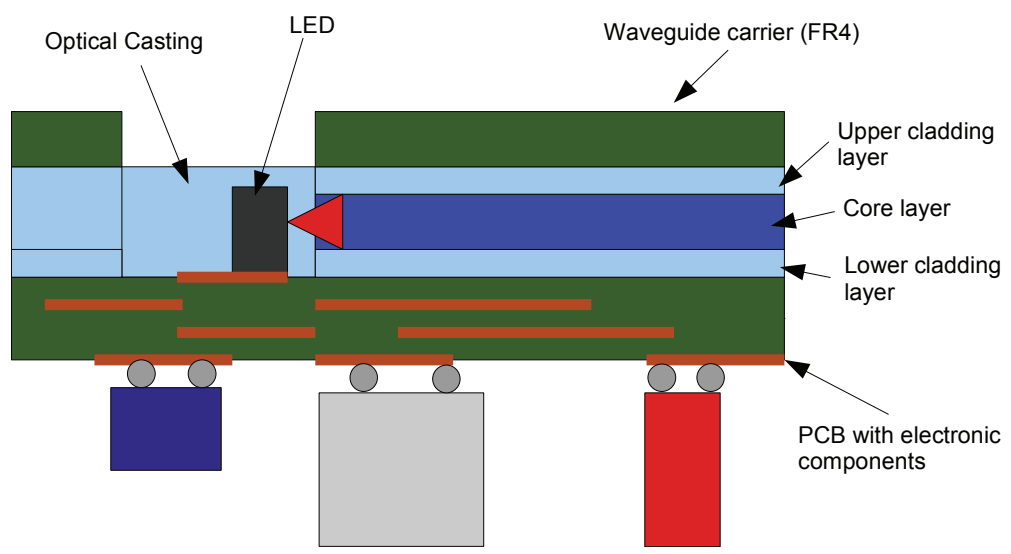

Fig. 6: Layer build-up of titration sensor

Fig. 6 shows the layer-build-up of the titration sensor. The base layer is the PCB with the assembled electronic components for the evaluation of the optical signals. The optical layer contains an upper- and a lower cladding which enclose the core layer containing the waveguide structures. Core and cladding consist of polymers with slightly different refractive indices, enabling the light-guiding effect. The eight LEDs are placed into cavities at the coupling positions. In order to optimize the optical coupling and for protection they are casted with an index matched adhesive. 


\subsection{Panel-Format Manufacturing of Optical Waveguide Structures}

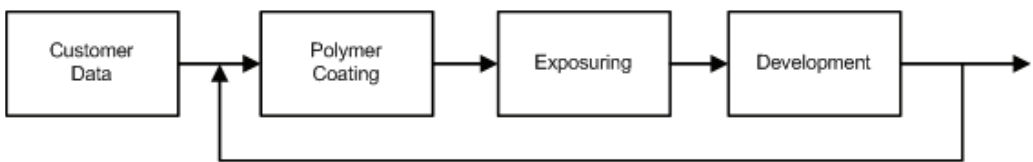

Fig. 7: Manufacturing process for electro-optical circuit boards

Fig. 7 shows the manufacturing process of the optical waveguide structures. The different polymer layers are deposited by doctor blading, which allows to level the very rough surface of the PCB-material. The process requirements for polymer deposition are quite demanding: $\pm 5 \mu \mathrm{m}$ of layer thickness variation are tolerated.

The exposure step is the main process of waveguide manufacturing. vario-optics ag uses two different technologies for exposure: either Laser Direct Imaging (LDI) or mask exposure. Mask exposure was chosen within this project, because it is very time efficient and allows the structuring of the complex optical system.

During the development of the polymer layers, the non-exposed and still liquid polymer material is washed away using appropriate chemicals. Key requirement for the lithographic process is the roughness of the side walls which should be below a tenth of the used carrier wavelength. Therefore, the development process is critical, because it significantly influences the attenuation of the waveguides.

Similar to PCB industry, the optical layers are produced in panel sizes of $460 \mathrm{~mm}$ x $305 \mathrm{~mm}$ (Fig. 8). Unfortunately, substrates of this size show extensive warping, which disturbs significantly the manufacturing processes. To flatten the substrates during the processing vacuum chucks were applied.
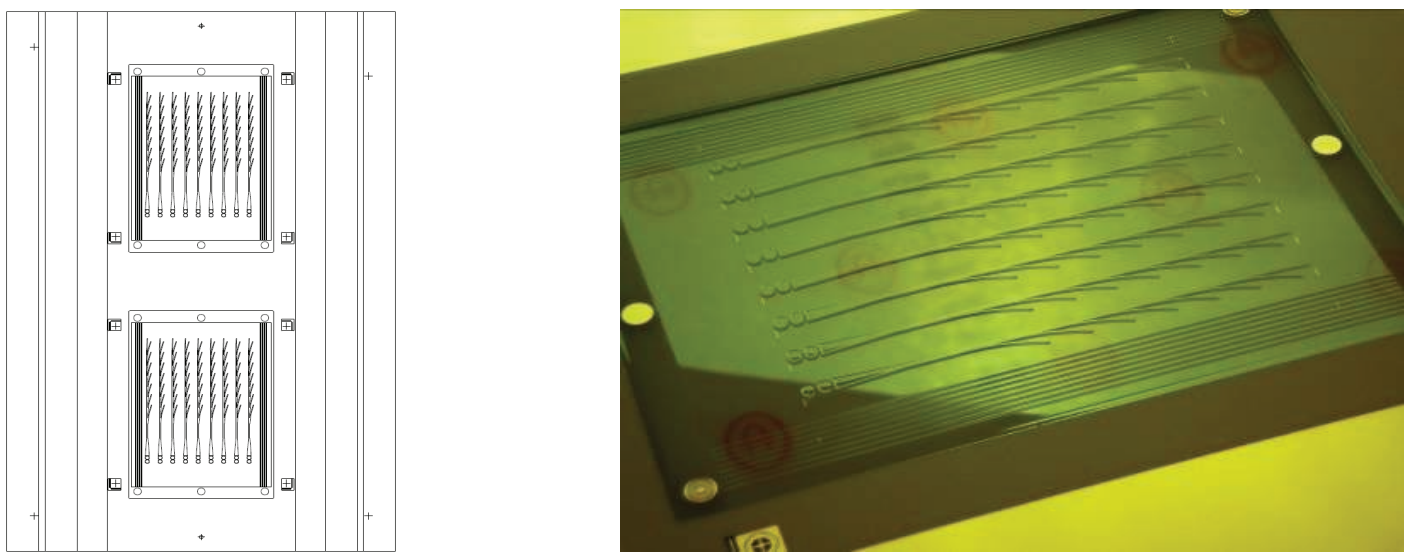

Fig. 8: Panel based manufacturing of electro-optical circuit board

To achieve sufficient coupling efficiencies with LEDs, light guides with cores heights of $500 \mu \mathrm{m}$ have to be used. Conventional EOCBs for datacom applications consist of light guides with a height of only $50 \mu \mathrm{m}$. Thus the manufacturing process of the light guides had to be optimized to meet the requirements like shape accuracy, side-wall roughness and absorption.

First tests showed a massive side-wall roughness for the waveguide cores (Fig. 9a). This led to unacceptable stray-losses. After an extensive process development, optimized manufacturing parameters were found that resulted in significantly reduced side-wall roughnesses and stray losses (Fig. 9b).
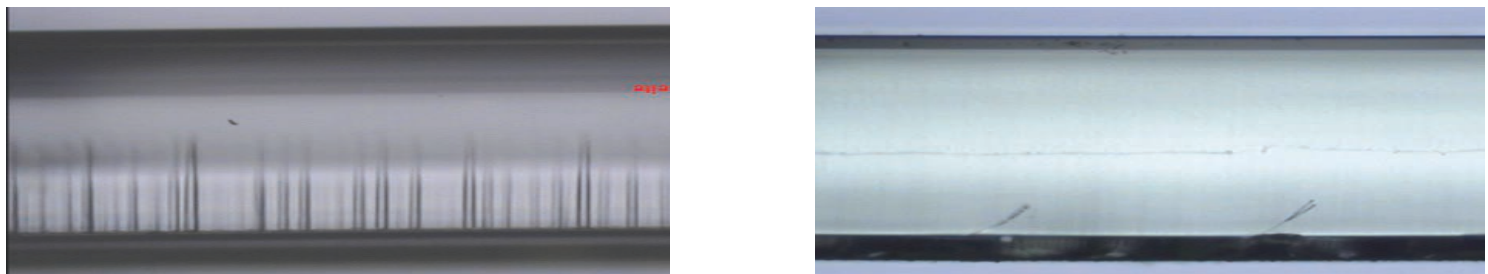

Fig. 9: Side-wall roughness: a) before optimization b) after optimization of the manufacturing parameters 
Evaluation and Results

During the last phase of the project, test measurements (titrations) were performed to verify the operational reliability of the sensor. It could be shown that the achieved irradiance produced on the detector has a light-dark contrast, which allows titrations in daylight or artificial light, with a reliable detection of colour changes of indicators, without additional light shields.

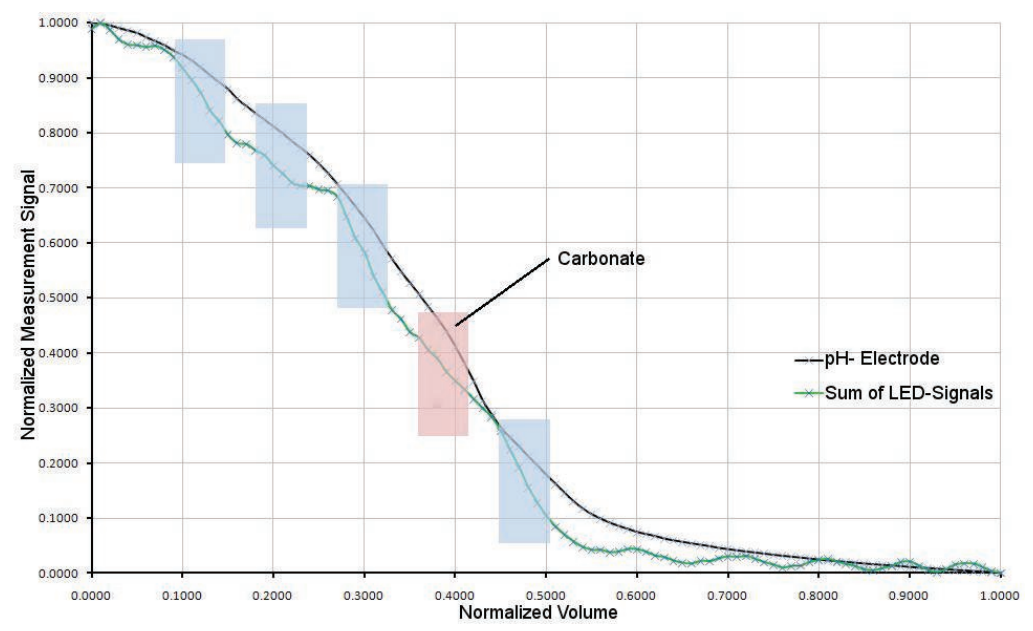

Fig. 10: Titration curve of a 4-acid mixture, titrated with sodium hydroxide. Indicator is a mixture of universal indicators from $\mathrm{pH}$ 0-5/4-10/9-13.

The novel sensor module proves the ability to determine multiple parameters of a titration simultaneously. It allows to determine a 4-acid mixture (pK value in monotonic distances) with only one indicator mixture in a single titration run (see Fig. 10). Furthermore, the titration speed can be set much faster than in a classical titration. Various tests showed that it is limited by the homogenous mixture of the liquids and not by the sensor system. The selection of an appropriate indicator is also no longer of great importance. This is due to the fact that the sensor covers the whole visual range in which the colour changes can occur. The sensor is already developed to the extent that the elaboration of appropriate control and evaluation algorithms are addressed.

\section{Conclusions}

A highly integrated titration sensor based on planar polymer waveguides has been successfully developed. The system is very cost efficient because of mask-based photolithographic manufacturing of the waveguide structures. The very short evaluation time of the sensor allows the measurement of titration of 4-acid mixtures processes at once, in realtime and with only one indicator mixture.

\section{Acknowledgement}

This project was kindly supported by the Swiss Commission for Technology and Innovation (CTI) .

\section{References}

[1] G. Schulze and J. Simon, Maßanalyse: Theorie und Praxis der Titrationen mit chemischen und physikalischen Indikationen, Gruyter, 2009.

[2] Y. Uematsu, T. Ozeki, and Y. Unno, "Efficient power coupling between an MH LED and a taper-ended multimode fiber," IEEE Journal of Quantum Electronics, vol. 15, 1979, pp. 86-92.

[3] M.C. Hudson, "Calculation of the Maximum Optical Coupling Efficiency into Multimode Optical Waveguides," Applied Optics, vol. 13, Mai. 1974, pp. 1029-1033.

[4] T. Kurokawa, N. Takato, and Y. Katayama, "Polymer optical circuits for multimode optical fiber systems," Applied Optics, vol. 19, 1980, pp. 3124-3129.

[5] H. Naumann and G. Schröder, Bauelemente der Optik, Fachbuchverlag Leipzig, 1992.

[6] H. Ma, A. Jen, and L. Dalton, "Polymer-Based Optical Waveguides: Materials, Processing, and Devices," Advanced Materials, vol. 14, 2002, pp. 1339-1365.

[7] F. Betschon, M. Michler, D. Craiovan, M. Halter, K. Dietrich, J. Kremmel, J. Franke, M. Gmür, and S. Paredes, "Mass production of planar polymer waveguides and their applications," San Francisco, California, USA: 2010, pp. 76070M-76070M-14. 